\section{Response to: 'Role of tubulointerstitial injury in ANCA-associated vasculitis is underestimated' by Moiseev et al}

We thank Dr Moiseev et $a l^{1}$ for their letter about the role of tubulointerstitial injury in antineutrophil cytoplasmic antibody (ANCA)-associated vasculitis (AAV), as a response to our recently published article 'Urinary epidermal growth factor predicts renal prognosis in antineutrophil cytoplasmic antibody-associated vasculitis' ${ }^{2}$

Histologically, the typical feature in the kidneys of patients with AAV is pauci-immune necrotising crescentic glomerulonephritis with little immunoglobulin and complement deposition in the glomerular capillary walls. ${ }^{3}$ Theoretically, glomerular injury is the 'upstream' in ANCA glomerulonephritis, and tubulointerstitial injury is commonly thought to be the 'downstream' of glomerular injury. Therefore, current biomarkers for ANCA glomerulonephritis have been mainly focused on the glomerulus. However, it has been suggested that markers of tubulointerstitial lesions are also important for assessing disease severity and predicting renal prognosis in patients with different renal diseases, even those who have 'glomerulocentric' paradigm. Ju $e t a l^{4}$ found that urinary epidermal growth factor (EGF), as a specific tubular marker, could be a predictor of chronic kidney disease progression in patients with glomerular diseases. Moreover, the role of tubulointerstitial markers was confirmed in several studies in patients with different forms of glomerulonephritis, such as diabetic nephropathy, ${ }^{5} \operatorname{IgA}$ nephropathy $(\operatorname{IgAN})^{6}$ and lupus nephritis. ${ }^{7}$ Some studies suggested that interstitial inflammation, interstitial fibrosis and tubular atrophy may have predictive value in detecting the risk of disease progression in patients with $\mathrm{AAV}^{8}{ }^{9}$

Although corticosteroid and cyclophosphamide improve the outcome, many patients with AAV experience progression of renal injury despite long-term immunosuppressive therapy. However, there is lack of good tubulointerstitial markers for predicting the renal prognosis in AAV.

We concur with Moiseev et $a l^{1}$ that it is important to evaluate whether biomarkers of tubulointerstitial injury can help the clinical decision-making process for patients with AAV. In our study, we found that tubulointerstitial EGF mRNA expression was significantly associated with estimated glomerular filtration rate (eGFR) at time of biopsy in patients with AAV. Moreover, we found that the ratios of urinary EGF to creatinine (uEGF/ $\mathrm{Cr}$ ) were associated with more severe renal disease, renal resistance to treatment and higher risk of progression to composite outcome in patients with AAV. It should be noted that the predictive value of urinary EGF is not specific for AAV, and some studies showed that it has the ability to predict the development of diabetic nephropathy and IgAN. ${ }^{10} 11$

In conclusion, urinary EGF/Cr level may be a useful non-invasive biomarker to assess the degree of tubular damage and the potential for transition into chronic progressive kidney disease in patients with AAV. To evaluate the utility of urinary EGF for guiding AAV treatment decision-making, further larger and multicentre cohort studies will be needed.
Liang Wu, ${ }^{1,2,3,4}$ Min Chen ${ }^{1,2,3,4}$

${ }^{1}$ Renal Division, Peking University First Hospital, Beijing, China

${ }^{2}$ Institute of Nephrology, Peking University, Beijing, China

${ }^{3}$ Key Laboratory of Renal Disease, Ministry of Health of China, Beijing, China

${ }^{4}$ Key Laboratory of Chronic Kidney Disease Prevention and Treatment, Peking University, Beijing, China

Correspondence to Prof Min Chen, Renal Division, Peking University First Hospital, Peking University, Institute of Nephrology, Beijing 100034, China; chenmin74@sina.com

Handling editor Josef S Smolen

Contributors LW wrote the manuscript. MC revised the manuscript and approved the final manuscript.

Competing interests None declared.

Patient consent Not required.

Provenance and peer review Commissioned; internally peer reviewed.

(C) Author(s) (or their employer(s)) 2019. No commercial re-use. See rights and permissions. Published by BMJ.

\section{Check for updates}

To cite Wu L, Chen M. Ann Rheum Dis 2019;78:e112.

Received 25 July 2018

Accepted 27 July 2018

Published Online First 12 August 2018

\section{Linked}

- http://dx.doi.org/10.1136/annrheumdis-2018-214095

Ann Rheum Dis 2019;78:e112. doi:10.1136/annrheumdis-2018-214145

\section{REFERENCES}

1 Bulanov N, Chebotareva NV, Novikov PI, et al. Role of tubulointerstitial injury in ANCA-associated vasculitis is underestimated. Ann Rheum Dis 2018. doi: annrheumdis-2018-214095.

2 Wu L, Li XQ, Goyal T, et al. Urinary epidermal growth factor predicts renal prognosis in antineutrophil cytoplasmic antibody-associated vasculitis. Ann Rheum Dis 2018:doi: annrheumdis-2017-212578. [Epub ahead of print: 08 May 2018].

3 Chen M, Jayne DRW, Zhao MH. Complement in ANCA-associated vasculitis: mechanisms and implications for management. Nat Rev Nephro/ 2017;13:359-67.

4 Ju W, Nair V, Smith S, et al. Tissue transcriptome-driven identification of epidermal growth factor as a chronic kidney disease biomarker. Sci Trans/ Med 2015:7:316ra193.

5 Mise K, Hoshino J, Ueno T, et al. Prognostic Value of Tubulointerstitial Lesions, Urinary $\mathrm{N}$-Acetyl- $\beta$ - $d$-Glucosaminidase, and Urinary $\beta 2$-Microglobulin in Patients with Type 2 Diabetes and Biopsy-Proven Diabetic Nephropathy. Clin J Am Soc Nephrol 2016;11:593-601.

6 Alamartine E, Sauron C, Laurent B, et al. The use of the Oxford classification of $\lg A$ nephropathy to predict renal survival. Clin J Am Soc Nephrol 2011;6:2384-8.

7 Hsieh C, Chang A, Brandt D, et al. Predicting outcomes of lupus nephritis with tubulointerstitial inflammation and scarring. Arthritis Care Res 2011:63:865-74.

8 Ford SL, Polkinghorne KR, Longano A, et al. Histopathologic and clinical predictors of kidney outcomes in ANCA-associated vasculitis. Am J Kidney Dis 2014;63:227-35.

9 Hauer HA, Bajema IM, Van Houwelingen HC, et al. Determinants of outcome in ANCA-associated glomerulonephritis: a prospective clinico-histopathological analysis of 96 patients. Kidney Int 2002;62:1732-42.

10 Betz BB, Jenks SJ, Cronshaw AD, et al. Urinary peptidomics in a rodent model of diabetic nephropathy highlights epidermal growth factor as a biomarker for renal deterioration in patients with type 2 diabetes. Kidney Int 2016;89:1125-35.

11 Torres DD, Rossini M, Manno C, et al. The ratio of epidermal growth factor to monocyte chemotactic peptide- 1 in the urine predicts renal prognosis in $\lg \mathrm{A}$ nephropathy. Kidney Int 2008;73:327-33. 